We are given the conics $C_{1}$ and $C_{2}$, and $A$ is any one of their intersections. Take any point $P$ in the plane. Then lines through $P$ cut $C_{1}$ in pairs of points $U, V$ which form an involution on $C_{1}$. These pairs are projected from $A$ by a pencil of lines in involution whose pairs meet $C_{2}$ in the pairs of points $X, Y$ of an involution. Their joins $X Y$ must therefore pass through a fixed point $Q$.

Clearly, each line $P U V$ can only lead to the unique line $Q X Y$, and each $Q X Y$ can only be arrived at from a unique $P U V^{\gamma}$; in other words, the lines $P U V$ and $Q X Y$ are pairs of a $(1,1)$ correspondence.

Consider now lines through $P$ and $Q$ conjugate with respect to the conic $C_{2}$. They are seen to be pairs of another $(1,1)$ correspondence; for given any line $\alpha$ through $P$, its pole with respect to $C_{2}$, and therefore the line $\beta$ joining $Q$ to the pole, is uniquely determined, and starting from $\beta$ we come back uniquely to $\alpha$.

Now in general there are two lines through $P$ for which the corresponding lines of the pencil through $Q$ in the two $(1,1)$ correspondences coincide; that is, there are two lines through $P$ such that ( $K L X Y)$ is harmonic, in which case by projection from $A,(K L U V)$ is harmonic. Thus through a general point in the plane there pass two lines only which cut the two conics harmonically. It follows that such lines envelope a conic.

\title{
A Useful Expansion in Applications of Determinants
}

\author{
By A. C. Aitken.
}

The expansion, in the form of a series, of the quotient of two determinants, in which the numerator determinant differs from the denominator in one column only, is moderately well known. It was found in 1825 by F. Schweins (see Muir's History, Vol. 1, pp. 171-2), and has many times since been rediscovered or proved ad hoc for particular applications.

There is a special case of this expansion, so useful that it seems worth while to present it on its own account. It is, for example,

$\left|a_{1} b_{2} c_{3} d_{4}\right| \div\left|b_{2} c_{3} d_{4}\right|=a_{1}-\frac{a_{2} \cdot b_{1}}{1 . b_{2}}-\frac{\left|a_{2} b_{3}\right|\left|b_{1} c_{2}\right|}{b_{2}\left|b_{2} c_{3}\right|}-\frac{\left|a_{2} b_{3} c_{4}\right|\left|b_{1} c_{2} d_{3}\right|}{\left|b_{2} c_{3}\right|\left|b_{2} c_{3} d_{4}\right|}$ 
$\mathbf{x} \times \mathbf{v i}$

where determinants are denoted by their diagonal elements, e.g.

$$
\left|b_{2} c_{3} d_{4}\right| \equiv\left|\begin{array}{ccc}
b_{2} & b_{3} & b_{4} \\
c_{2} & c_{3} & c_{4} \\
d_{2} & d_{3} & d_{4}
\end{array}\right|
$$

The numerator is obtained by bordering the denominator by a leading row and column. We assume that the denominators in the expansion do not vanish. The general result is entirely similar to the particular case we have used for illustration.

Proof. Denoting the left side of (1) by $Q_{4}$, we have

$$
Q_{4}-Q_{3}=|| a_{1} b_{2} c_{3} d_{4}|| b_{2} c_{3} d_{4}|| \div\left\{\left|b_{2} c_{3}\right|\left|b_{2} c_{3} d_{4}\right|\right\}
$$

The compound determinant in the numerator on the right is the "extensional," by means of the "extension" $\left(b_{2} c_{3}\right)$, of

$$
\text { Hence its value is } \begin{aligned}
& -\left|a_{4} b_{2} c_{3}\right|\left|d_{1} b_{2} c_{3}\right| \\
= & -\left|a_{2} b_{3} c_{4}\right|\left|b_{1} c_{2} d_{3}\right|,
\end{aligned}
$$

by an even number of interchanges of rows and columns (letters and suffixes).

Adding now $Q_{4}-Q_{3}, Q_{3}-Q_{2}, Q_{2}-Q_{1}$ and $Q_{1}=a_{1}$, we have the desired result (1), and the proof of the general case is analogous.

Corollary. If $\left|a_{1} b_{2} c_{3} d_{4}\right|=0$, and $b_{2} \neq 0,\left|b_{2} c_{3}\right| \neq 0,\left|b_{2} c_{3} d_{4}\right| \neq 0$, we derive at once

$$
a_{1}=\frac{a_{2} \cdot b_{1}}{1 . b_{2}}+\frac{\left|a_{2} b_{3}\right|\left|b_{1} c_{2}\right|}{b_{2} \mid} \frac{\left|a_{2} b_{3} c_{4}\right|\left|b_{1} c_{2} d_{3}\right|}{\left|b_{2} c_{3}\right|\left|b_{2} c_{3} d_{4}\right|},
$$

the general result of this kind being similar.

\section{Applications.}

1. Interpolation. Let us consider a standard problem, namely; given $n+1$ values of a polynomial $f(x)$, for $x=a_{0}, a_{1}, a_{2}, \ldots, a_{n}$, to determine $f(x)$ in terms of these values.

By way of illustration, let us find what polynomial $f(x)$ of the 3rd degree takes the values $f\left(a_{0}\right), f\left(a_{1}\right), f\left(a_{2}\right), f\left(a_{3}\right)$.

If we put $f(x)=c_{0}+c_{1} x+c_{2} x^{2}+c_{3} x^{3}$, this equation is consistent with four others obtained from it by putting $x=a_{0}, a_{1}, a_{2}, a_{3}$. Hence the eliminant (an alternant)

$$
\left|f(x) \quad a_{0}^{0} \quad a_{1}^{1} \quad a_{2}^{2} \quad a_{3}^{3}\right|=0
$$


Hence, by the Corollary (2) above, we have

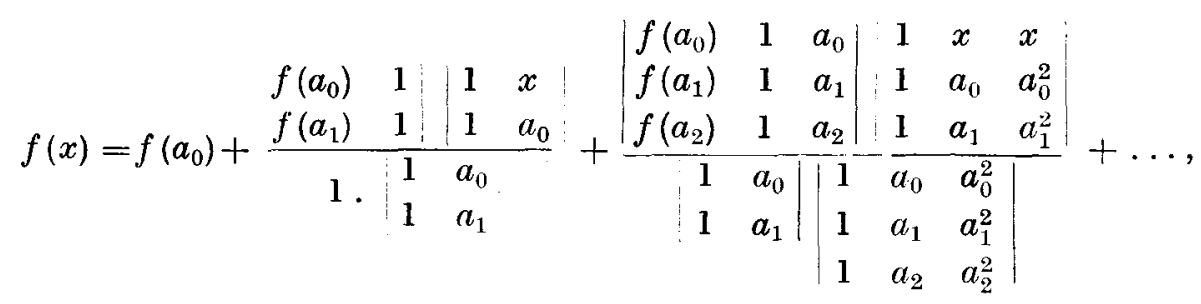

$$
\begin{aligned}
& =f\left(a_{0}\right)+\left(x-a_{0}\right) f\left(a_{0}, a_{1}\right)+\left(x-a_{0}\right)\left(x-a_{1}\right) f\left(a_{0}, a_{1}, a_{2}\right) \\
& +\left(x-a_{0}\right)\left(x-a_{1}\right)\left(x-a_{2}\right) f\left(a_{0}, a_{1}, a_{2}, a_{3}\right) \text {, }
\end{aligned}
$$

since factors cancel out from those alternants that are differenceproducts, and the other quotients of alternants in each term give Newton's divided differences, usually written $f\left(a_{0}, a_{1}\right), f\left(a_{0}, a_{1}, a_{2}\right)$, etc.

Thus we obtain Newton's divided difference formula of interpolation as a special case of our expansion. Lagrange's formula, on the other hand, is obtained by expanding the eliminant in (3) in terms of the elements of its first column.

2. Reduction of a Quadratic Form to a Sum of Squares.

It is well known that a non-degenerate quadratic form can be expressed by suitably bordering the adjugate of its determinant; for example,

$$
a x^{2}+b y^{2}+c z^{2}+2 h x y+2 g x z+2 f y z=-\Delta\left|\begin{array}{cccc}
0 & x & y & z \\
x & A & H & G \\
y & H & B & F \\
z & G & F & C
\end{array}\right| \div\left|\begin{array}{ccc}
A & H & G \\
H & B & F \\
G & F & C
\end{array}\right|,
$$

where capital letters denote, as usual, cofactors, and the denominator is not zero.

If further the leading minors of this denominator do not vanish, we may apply our expansion (1) and obtain the quadratic form in the shape

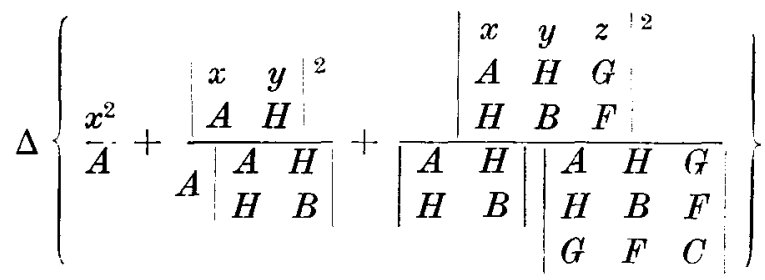

$$
\begin{aligned}
& =\Delta x^{2} / A+\left|\begin{array}{cc}
x & y \\
A & H
\end{array}\right|^{2} /(c A)+(g x+f y+c z)^{2} / c,
\end{aligned}
$$




\section{xxviii}

by Jacobi's theorem on a minor of the adjugate. This is really Lagrange's reduction (1759) of a quadratic form to a sum of squares. Incidentally, if $c, A$ and $\Delta$ (minors each contained boxlike in its successor) are all positive, the coefficients of the three squared expressions in the reduced quadratic above are all positive. This again, with the aid of Sylvester's theorem on the "Inertia of Quadratic Forms," gives a classic result, namely, that if a set of overlapping minors of successive orders in $\Delta$, and finally $\Delta$ itself, are all positive, this ensures that the quadratic form shall be positive definite.

\section{Problems of Approximation by Least Squares.}

Let us next consider the problem of representing an arbitrary function $u(x)$ as closely as possible over the range $x=a$ to $x=b$ by a polynomial $f(x)$ of assigned degree, the criterion of approximation being that the integral of squared deviations

$$
S^{2}=\int_{a}^{b} w(x)\{f(x)-u(x)\}^{2} d x
$$

shall be a minimum. The function $w(x)$ is a "weight function," positive over at least part of the range. By way of illustration, let us find such a polynomial of the 3rd degree.

Let

$$
\begin{gathered}
f(x)=c_{0}+c_{1} x+c_{2} x^{2}+c_{3} x^{3} \\
\int_{a}^{b} x^{r} w(x) d x=m_{r}, \int_{a}^{b} x^{r} w(x) u(x) d x=M_{r} .
\end{gathered}
$$

Now (5) and the minimal conditions $\quad \frac{1}{2} \partial S^{2} / \partial c_{r}=0$, being consistent, give the eliminant

$$
\left|\begin{array}{ccccc}
f(x) & 1 & x & x^{2} & x^{3} \\
M_{0} & m_{0} & m_{1} & m_{2} & m_{3} \\
M_{1} & m_{1} & m_{2} & m_{3} & m_{4} \\
M_{2} & m_{2} & m_{3} & m_{4} & m_{5} \\
M_{3} & m_{3} & m_{4} & m_{5} & m_{6}
\end{array}\right|=0
$$

Applying our Corollary (2), we obtain

$$
f(x)=\frac{M_{0}}{m_{0}}+\frac{\left|\begin{array}{cc}
M_{0} & m_{0} \\
M_{1} & m_{1}
\end{array}\right|\left|\begin{array}{cc}
1 & x \\
m & m_{1}
\end{array}\right|}{m_{0}\left|\begin{array}{ll}
m_{0} & m_{1} \\
m_{1} & m_{2}
\end{array}\right|}+\frac{\left|\begin{array}{lll}
M_{0} & m_{0} & m_{1} \\
M_{1} & m_{1} & m_{2} \\
M_{2} & m_{2} & m_{3}
\end{array}\right|\left|\begin{array}{ccc}
1 & x & x^{2} \\
m_{0} & m_{1} & m_{2} \\
m_{1} & m_{2} & m_{3}
\end{array}\right|}{\left|\begin{array}{lll}
m_{0} & m_{1} \\
m_{1} & m_{2}
\end{array}\right| \begin{array}{lll}
m_{0} & m_{1} & m_{2} \\
m_{1} & m_{2} & m_{3} \\
m_{2} & m_{3} & m_{4}
\end{array} \mid}+\ldots(
$$


Thus $f(x)$ has been expressed in terms of a set of polynomials

$$
\mathbf{1},\left|\begin{array}{cc}
1 & x \\
m_{0} & m_{1}
\end{array}\right|, \quad\left|\begin{array}{ccc:c:cccc}
1 & x & x^{2} & 1 & x & x^{2} & x^{3} \\
m_{0} & m_{1} & m_{2} \\
m_{1} & m_{2} & m_{3} & m_{0} & m_{1} & m_{2} & m_{3} \\
m_{1} & m_{2} & m_{3} & m_{4} \\
m_{2} & m_{3} & m_{4} & m_{5}
\end{array}\right|,
$$

depending on $w(x)$, but not on $u(x)$.

It is a simple matter to prove that these polynomials satisfy weighted orthogonal conditions under integration over the range, namely

$$
\int_{a}^{b} x^{r} w(x) P_{s}(x) d x=0, \quad r<s
$$

Certain special cases are interesting. For example if $w(x)=1$ the polynomials are Legendre polynomials. We may also apply the same method to discrete data $u(x)$, summation taking the place of integration.

The above are only a few of many situations in which the expansions to which we have drawn attention serve at least to discover the formal solution of a problem, and often something more. 